ARTICLE

\title{
Decoupling the origins of irreversible coulombic efficiency in anode-free lithium metal batteries
}

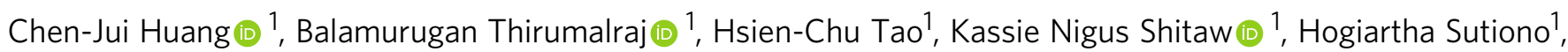
Tesfaye Teka Hagos², Tamene Tadesse Beyene', Li-Ming Kuo ${ }^{1}$, Chun-Chieh Wang ${ }^{3}$, She-Huang Wu², Wei-Nien Su (i) ${ }^{2}$ \& Bing Joe Hwang (10) 1,3,4凶

Anode-free lithium metal batteries are the most promising candidate to outperform lithium metal batteries due to higher energy density and reduced safety hazards with the absence of metallic lithium anode during initial cell fabrication. In general, researchers report capacity retention, reversible capacity, or rate capability of the cells to study the electrochemical performance of anode-free lithium metal batteries. However, evaluating the behavior of batteries from limited aspects may easily overlook other information hidden deep inside the meretricious results or even lead to misguided data interpretation. In this work, we present an integrated protocol combining different types of cell configuration to determine various sources of irreversible coulombic efficiency in anode-free lithium metal cells. The decrypted information from the protocol provides an insightful understanding of the behaviors of LMBs and AFLMBs, which promotes their development for practical applications.

\footnotetext{
${ }^{1}$ Department of Chemical Engineering, National Taiwan University of Science and Technology, Taipei, Taiwan. ${ }^{2}$ Graduate Institute of Applied Science and Technology, National Taiwan University of Science and Technology, Taipei, Taiwan. ${ }^{3}$ National Synchrotron Radiation Research Center (NSRRC), Hsinchu, Taiwan.

${ }^{4}$ Sustainable Energy Development Center, National Taiwan University of Science and Technology, Taipei, Taiwan. ${ }^{凶}$ email: bjh@mail.ntust.edu.tw
} 
ithium metal, with an ultrahigh theoretical specific capacity $\left(3860 \mathrm{mAh}^{-1}\right)$ and low redox potential $(-3.040 \mathrm{~V}$ vs. standard hydrogen electrode), has already been extensively investigated over the four decades ${ }^{1,2}$. However, lithium metal batteries (LMB) still suffer from several barriers and yet to be commercialized. More specifically, the safety issues induced by $\mathrm{Li}$ dendrite growth and internal short circuit (ISC) ${ }^{3}$, poor efficiency attributed to the formation of high surface area lithium (HSAL, dendrite) and dead $\mathrm{Li}^{4,5}$, and severe electrolyte decomposition at the negative electrode leading to electrolyte dry-up and the formation of thick solid electrolyte interphase (SEI) that increases the internal resistance and consumes the electrolytes ${ }^{6-11}$.

To overcome the aforementioned challenges, one has to systematically study Li metal stability/protection ${ }^{12}$, SEI formation mechanism ${ }^{13,14}$, and suppression of $\mathrm{Li}$ dendrite growth in $\mathrm{LMB}^{15-17}$. Many works have also been done by using different electrolyte formulas ${ }^{18}, 3 \mathrm{D}$ architecture $\mathrm{Li}^{19}$, and artificial coating layers $^{20}$ to study their effect on increasing the electrochemical performance of LMB. Meanwhile, several key factors would still affect the cycling performance of LMB and are crucial in achieving high specific energy of $500 \mathrm{Wh} \mathrm{kg}^{-1}$ demanded by electric vehicle energy-storage market such as electrolyte amount $^{21}$, temperature ${ }^{22}$, pressure ${ }^{23}$, amount of $\mathrm{Li}$ or cahode $^{24,25}$, and current density applied ${ }^{9}$, etc. Recently, anodefree lithium metal batteries (AFLMBs) are considered as phenomenal energy-storage systems owing to higher energy density than that of LMB, which with excess Li in the system, and greatly reduced safety risks since no Li metal is used during cell manufacturing, which remarkably increases the simplicity of cell fabrication and reduces the cost of cell assembly, too ${ }^{26-31}$.

However, in most of the published works, the electrochemical performance of LMBs/AFLMBs is often discussed by comparing capacity retention, reversible capacity, or rate capability, which easily overlooks or even misunderstands the information that is concealed by the meretricious results when adopting only one or two points of view. To systematically evaluate the electrochemical performance of both LMBs and AFLMBs, and unfold all the messages hidden within the battery, one has to comprehensively examine the information from all the possible perspectives. More importantly, integrating all the unraveled phenomena and messages to have a better overall evaluation of the battery systems is essential. One efficient way is to study the irreversible coulombic efficiency
(irr-CE), which may represent the side reactions and sources of capacity loss in the battery. Meng et al. ${ }^{32}$ demonstrated an analytical method of titration gas chromatography (TGC) to quantify the contribution of dead $\mathrm{Li}$ to the total irr-CE in Li/Cu cells, identifying dead $\mathrm{Li}$ as the major reason accounted for the capacity loss of $\mathrm{Li} / \mathrm{Cu}$ cells. They comprehensively discussed the formation of inactive $\mathrm{Li}$ and determined the origin of irr-CE of $\mathrm{Li}$ anodes within $\mathrm{Li} / \mathrm{Cu}$ cells by decoupling the dead $\mathrm{Li}$ and SEI formation, which provides strategies for more-efficient $\mathrm{Li}$ plating and stripping on $\mathrm{Cu}$ substrate. However, the important information of the irr-CE or capacity loss from the cathode and cross-talk effects in cathode/Li or anode-free cathode/ $\mathrm{Cu}$ cells could not be extracted from the TGC method. Thus, there is still a lack of holistic methodology to identify and quantify the irr-CEs in LMBs and AFLMBs.

In this work, we systematically study four different types of cell configuration, including $\mathrm{Li} / \mathrm{Li}$ symmetric cells ${ }^{33}, \mathrm{Li} / \mathrm{Cu}$ cells, cathode/Li cells, and cathode/Cu anode-free cells, as an integrated protocol to unfold the intrinsic reasons and contributions of individual irr-CEs from not only $\mathrm{Li}$ anodes in $\mathrm{Li} / \mathrm{Cu}$ cells, but also the cathodes in cathode/Li half-cells. Furthermore, the cause of low irr-CE of AFLMBs can also be determined at different states of the batteries through the proposed protocol. Meanwhile, we also observed dendritic Li induced ISC and visualize the formation of dead $\mathrm{Li}$ in a $\mathrm{Li} / \mathrm{Cu}$ cell using in situ optical microscopy (OM) and transmission X-ray microscopy (TXM), and proposed the mechanism of $\mathrm{Li}$ nucleation and deposition/dissolution on $\mathrm{Cu}$. This work provides an overall understanding and quantification to the irr-CEs from the full spectrum of different cell configurations such as first extra SEI, dead Li and subsequent SEI, cross-talk effects, first cycle intrinsic irreversible capacity of the cathode, and subsequent oxidative electrolyte decomposition. The proposed protocol could serve as a platform from an overall perspective to evaluate the performance of LMBs and AFLMBs and can be widely applied to various systems for the development of nextgeneration high-energy batteries.

\section{Results}

In situ OM observation of $\mathrm{Li}$ deposition/dissolution. $\mathrm{Li}$ deposition/dissolution upon cycling with the subsequent dendrite growth and formation of dead $\mathrm{Li}$ are observed by in situ OM in Fig. 1a (Supplementary Movie 1, 2, and 3). In the beginning, both
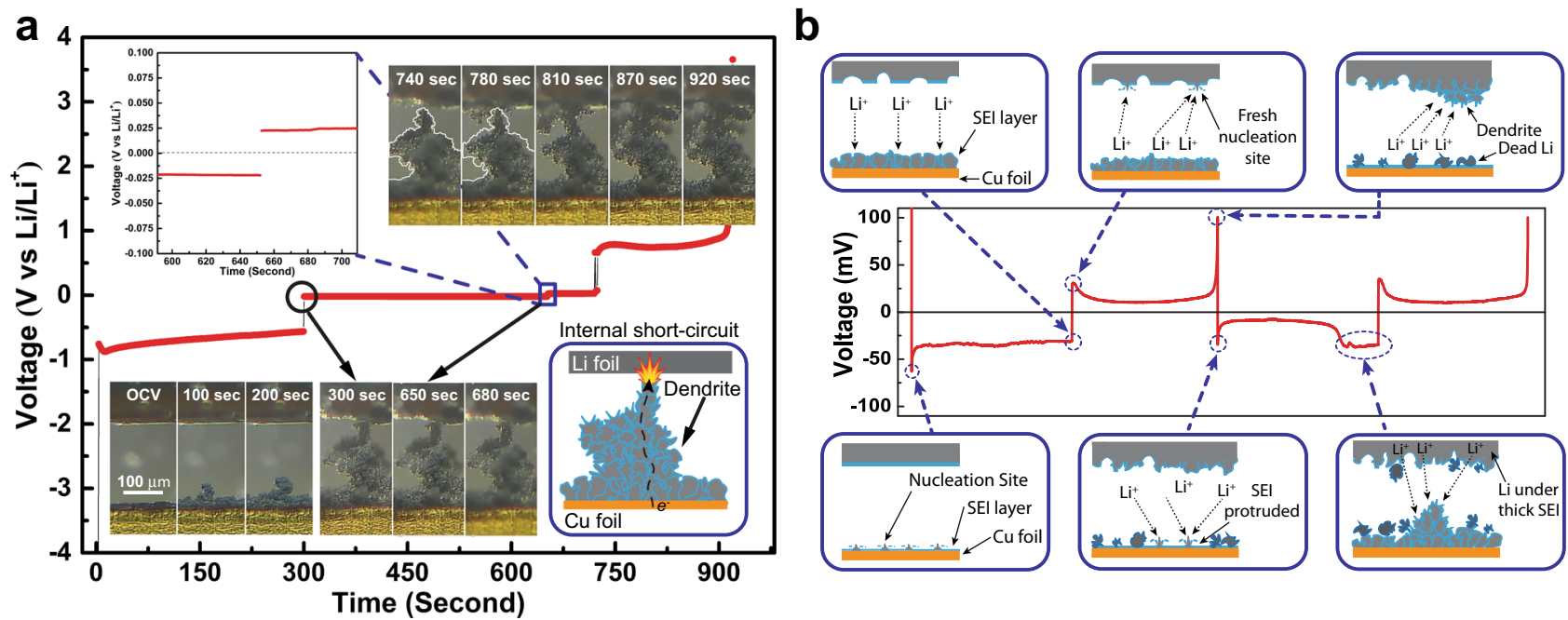

Fig. 1 In situ OM and Li deposition/dissolution scheme. a In situ OM measurement of Li deposition/dissolution on Cu electrode under an ultrahigh current density of $500 \mathrm{~mA} \mathrm{~cm}^{-2}$ for demonstration of short-circuiting. It should be noted that the observed short circuit region is different from the initial observation region for Li-plating process. b Scheme of Li deposition/dissolution on $\mathrm{Cu}$ foil during cycling of Li/Cu cell under a current density of $0.2 \mathrm{~mA} \mathrm{~cm}^{-2}$. 
$\mathrm{Cu}$ and $\mathrm{Li}$ surfaces are very smooth. In the first $10 \mathrm{sec}$ of plating, there was a clear potential drop observed from the curve, which was owing to the overpotential induced by the initial nucleation of Li on $\mathrm{Cu}$ foil and SEI fracture. After the nucleation sites were formed, the polarization was found reduced. The plated $\mathrm{Li}$ was homogeneous and dense during the first $100 \mathrm{sec}$; however, granular Li started to grow on different spots of $\mathrm{Cu}$ foil owing to the inhomogeneous plating of Li. After the initial granular Li emerged, which served as a fresh nucleus, HSAL vigorously grew on top of the granule rather than the compact and uniform Li beside it on $\mathrm{Cu}$ foil. In addition, non-uniform dissolution of $\mathrm{Li}$ and formation of a rough surface at the Li electrode were also observed during the first $300 \mathrm{sec}$, induced from the locally heterogeneous current distribution, which also accelerated the formation of dendritic $\mathrm{Li}$ on the $\mathrm{Cu}$ electrode (Supplementary Movie 1).

As the plating process continued, the HSAL eventually contacted the $\mathrm{Li}$ electrode and short-circuits occurred after $300 \mathrm{sec}$ of $\mathrm{Li}$ plating. Then, the cell voltage was found suddenly lifted to -0.022 $\mathrm{V}$ vs. $\mathrm{Li} / \mathrm{Li}^{+}$but not zero, indicating there is an SEI resistance between two contacted electrodes. Although the potentiostat was still applying negative current, no extra $\mathrm{Li}$ was plated on the working electrode during the short circuit. When the cell was switched to a stripping mode at $650 \mathrm{sec}$, the slightly positive cell voltage of $0.022 \mathrm{~V}$ vs. $\mathrm{Li}^{\prime} / \mathrm{Li}^{+}$was also observed in the stripping process owing to the SEI resistance between both contacted electrodes as in the deposition process. Besides, the morphology of the dendrite was kept unchanged without Li deposition/dissolution during the short circuit (Supplementary Movie 2).

After $740 \mathrm{sec}$ at the stripping process, the short-circuited HSAL between two electrodes suddenly disconnected and started to dissolve. However, dead Li was discovered after $40 \mathrm{sec}$ of stripping as the HSAL stopped dissolving owing to the higher charge resistance, leaving a large amount of dead $\mathrm{Li}$ on the $\mathrm{Cu}$ surface at the end of the stripping process. This results in poor CE observed from the cycling performance of the cell (Supplementary Movie 3).

Li nucleation and deposition/dissolution mechanism. Based on the OM (Fig. 1a) and TXM (Supplementary Fig. 1, Supplementary Movie 4 \& 5) observation of Li plating/stripping, the electrochemical phenomena of $\mathrm{Li}$ plating/stripping on $\mathrm{Cu}$ are investigated, and the corresponding proposed mechanism is shown in Fig. 1b. During the very beginning of Li deposition on $\mathrm{Cu}$ foil, an initial energy barrier needs to be overcome by first forming nucleation sites on the clean $\mathrm{Cu}$ surface, which causes the initial higher overpotential seen from the curve. Then, Li underneath the thick SEI layer on Li foil starts to dissolve and deposit onto the nucleation sites on the Cu surface, accompanied by the decrease of overpotential. Next, in the Li-stripping process from the $\mathrm{Cu}$ surface, the overpotential is induced from the resistance of the SEI layer protruding and fresh Li growth on the $\mathrm{Li}$ surface. At the end of the Li-stripping process from the $\mathrm{Cu}$ surface, the cell voltage suddenly lifted due to the total consumption of active $\mathrm{Li}$, leaving some dead $\mathrm{Li}$ at the $\mathrm{Cu}$ surface. From our previous in-operando TXM results ${ }^{4}$, we observed that the formation of dead $\mathrm{Li}$ is generally from the outer part of deposited $\mathrm{Li}$, which disconnects with the active $\mathrm{Li}$ at the electrode surface, similar to the observation in this work, and the stacking of dead $\mathrm{Li}$ is also observed after several plating/stripping processes. At the same time, on the other side, dendrites start to evolve on the roughened Li surface owing to non-uniform current distribution from the previous dissolution process. In the following cycles, the deposition/dissolution of $\mathrm{Li}$ is similar to the first cycle in general, including SEI fracture, fresh Li nucleation, dendrite growth, and dead $\mathrm{Li}$ formation at both sides. More detail regarding the SEI fracture mechanism is discussed in our previous work $^{34}$. However, it is worth noted that in the latter part of $\mathrm{Li}$ deposition in the second cycle, a second plateau with the potential similar to the deposition overpotential in the first cycle appeared. It is suggested that the first and second plateau is ascribed to the freshly deposited Li (HSAL) stripping and the bulk (original) Li, which is covered by the thick SEI owing to the low reduction potential of metallic $\mathrm{Li}$, stripping on the $\mathrm{Li}$ side, respectively. Thus, the overpotential relates to $\mathrm{Li}$ deposition/dissolution processes on the Li surface is significantly influenced by the nature of SEI, the HSAL on both $\mathrm{Cu}$, Li surfaces, and the charge transfer processes at both interfaces.

Proposed integrated protocol. In this section, the four types of cell configuration as an integrated protocol assembled by $\mathrm{Li} / \mathrm{Li}$ symmetric cell, $\mathrm{Li} / \mathrm{Cu}$ cell, cathode/ $\mathrm{Li}$ cell, and cathode/Cu anodefree cell was applied to comprehensively evaluate the performance of LMBs/AFLMBs and unravel the unseen messages concealed in the individual candidates. Figure 2 shows the charge/discharge profiles of all the cell configuration used in the protocol. Figure 3 shows the schematics of each cell configuration at its fully charged and discharged state with the scheme of the proposed protocol from both irr-CE and capacity perspective. By applying the integrated protocol, one can decrypt different information from each cell set-ups and dissect the origins of irr-CE and capacity lost within LMB/AFLMB quantitatively.

$\mathrm{Li} / \mathrm{Li}$ symmetric cell, with its charge/discharge curves shown in Fig. $2 \mathrm{a}$, is a useful configuration that allows us to understand the kinetics of $\mathrm{Li}$ deposition/dissolution by extracting the initial nucleation overpotential and polarization information ${ }^{12,33,35}$. It is also a powerful tool to investigate ISC, critical current density phenomenon, or SEI polarization on Li surface, which although depends on the surface quality of both Li electrodes. However, irr-CE is unable to be obtained from $\mathrm{Li} / \mathrm{Li}$ cell since the $\mathrm{CE}$ is always $\sim 100 \%$ due to excess $\mathrm{Li}$ from both $\mathrm{Li}$ electrodes that compensate the formation of dead $\mathrm{Li}$ and active Li lost from reductive electrolyte decomposition (SEI formation or gas generation). As a result, the consumption of active $\mathrm{Li}$ owing to the aforementioned reactions is invisible; thus, seldom discussed and often overlooked in this cell configuration ${ }^{4}$. Generally, it is not easy to report reliable and reproducible information on cycling performance or even ISC of this $\mathrm{Li} / \mathrm{Li}$ protocol unless the quality of Li surface employed, the amounts of electrolytes, and the pressure on the tested cell are well-controlled.

However, by replacing $\mathrm{Li}$ with $\mathrm{Cu}$ as the working electrode in $\mathrm{Li} / \mathrm{Cu}$ cell as shown in Fig. $2 \mathrm{~b}$, it is possible to quantify the inactive $\mathrm{Li}$ on the $\mathrm{Cu}$ electrode from the irr-CE of the $\mathrm{Li} / \mathrm{Cu}$ cell in each cycle. Figures $3 \mathrm{a}$ and $3 \mathrm{~d}$ show the scheme of $\mathrm{Li} / \mathrm{Cu}$ cell at fully plated and stripped state in the first cycle. In the $\mathrm{Li} / \mathrm{Cu}$ cell, as the excess $\mathrm{Li}$ is from the $\mathrm{Li}$ electrode, resulting in the $\mathrm{Cu}$ side as the limiting electrode. As there is no excessive metallic $\mathrm{Li}$ on $\mathrm{Cu}$ as it was in $\mathrm{Li} / \mathrm{Li}$ cell to compensate for the irreversible consumption of active $\mathrm{Li}$ on $\mathrm{Cu}$. The irreversible phenomena observed mainly reflect the behaviors of the $\mathrm{Cu}$ electrode. In general, the irr-CE in $\mathrm{Li} / \mathrm{Cu}$ cells can be separated into two main sources, namely dead Li and SEI formation. In particular, the irr$\mathrm{CE}$ is normally higher in the first cycle of $\mathrm{Li} / \mathrm{Cu}$ owing to the initial extra SEI formation on the $\mathrm{Cu}$ surface, causing a larger irr$\mathrm{CE}$ than that in the subsequent cycles, denoted as the first extra SEI (red bar in Fig. 3g). Thus, we proposed that the irr-CE of Li/ $\mathrm{Cu}$ cell in the first cycle contains both first extra SEI formation and dead Li+ sub. SEI (green bar in Fig. 3g). We have combined the contribution of dead $\mathrm{Li}$ and subsequent SEI owing to the fact that it is not possible to easily separate the fraction of which unless an experimental method like the TGC method is performed, and the fraction of subsequent SEI is not stable in 

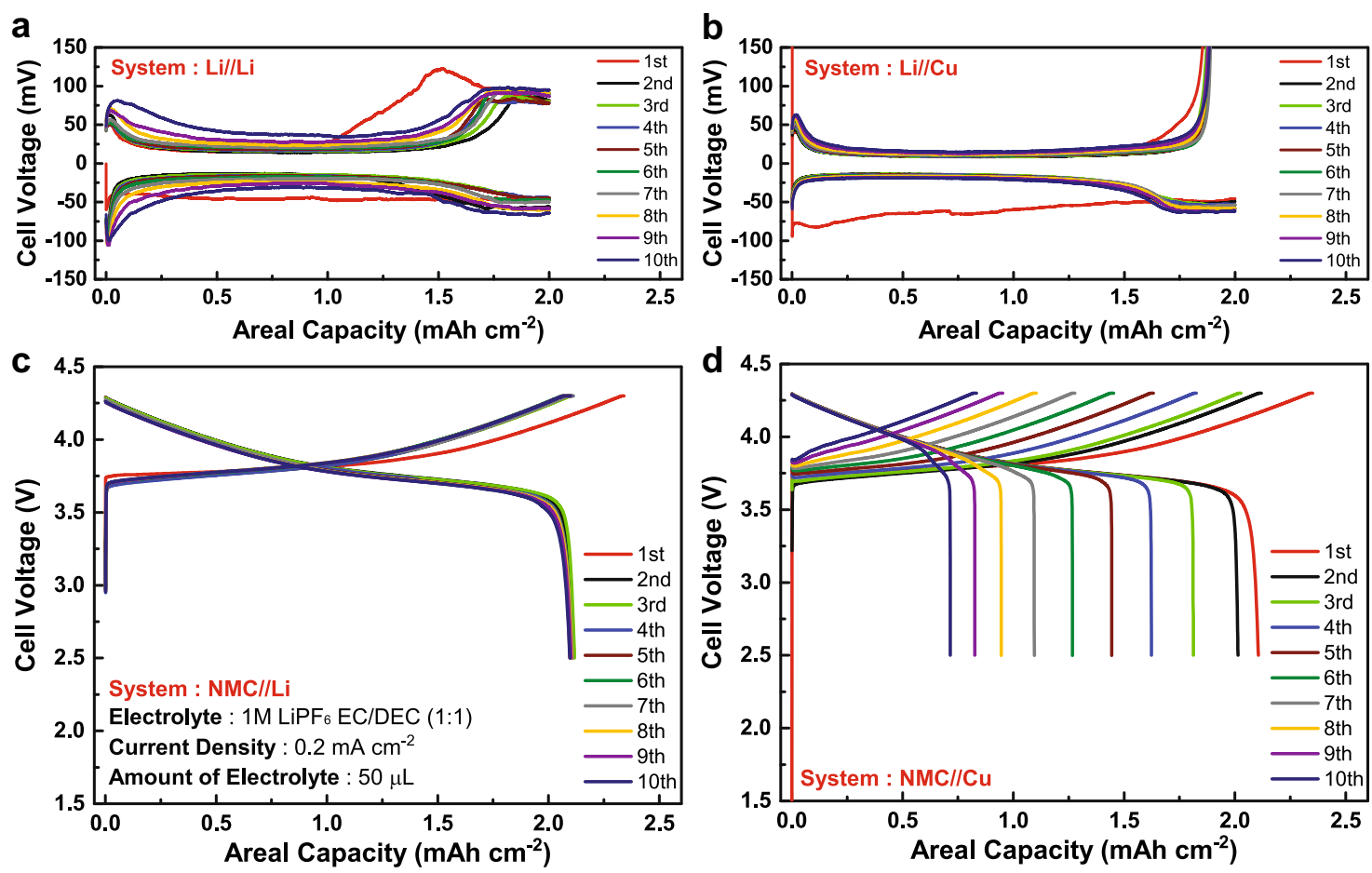

Fig. 2 Electrochemical charge and discharge curves. a Li/Li. b Li/Cu. c NMC/Li. d NMC/Cu. All the cells use 1 M LiPF 6 in EC:DEC (1:1) as electrolyte with the current density of $0.2 \mathrm{~mA} \mathrm{~cm}{ }^{-2}$. We selected NMC-111 as the cathode material used in this electrolyte. One can substitute the desired cathode material for cathode study in cathode/Li cell and AFLMB.

the subsequent cycles owing to the dendritic Li caused fracture ${ }^{34}$. Therefore, the irr-CE originated from the first SEI formation (red bar in Fig. $3 \mathrm{~g}$ ) can be calculated by subtracting the value in the first cycle with that of the second cycle from the $\mathrm{Li} / \mathrm{Cu}$ cell protocol. To conclude, the $\mathrm{Li} / \mathrm{Cu}$ cell is a useful protocol to provide reliable information for the study of electrolyte ${ }^{27,28,31}$, and surface engineering approaches ${ }^{29,36,37}$ for mitigating the irr$\mathrm{CE}$ ascribed to dead $\mathrm{Li}$ and SEI formation.

The third protocol is a cathode/Li cell, and the charge/discharge curves are shown in Fig. 2c, namely a half-cell for studying phenomena taking place at the cathode. When the cathode/Li cell is fully charged, $\mathrm{Li}^{+}$is de-intercalated from NMC and plated onto the $\mathrm{Li}$ anode along with the formation of dendritic or mossy $\mathrm{Li}$ (Fig. 3b). In reverse, $\mathrm{Li}^{+}$is stripped from the $\mathrm{Li}$ anode with some dead Li left on it and intercalated back into NMC (Fig. 3e). However, as there is a significant amount of active $\mathrm{Li}$ on the $\mathrm{Li}$ electrode compared with that in the cathode electrode, i.e., the capacity ratio of the anode to cathode $(\mathrm{A} / \mathrm{C})$ is $>1$ and cathode is the limiting electrode, the excess metallic $\mathrm{Li}$ will compensate the active $\mathrm{Li}$ loss due to dead $\mathrm{Li}$ formation and reductive electrolyte decomposition at the anode side, leading them invisible from the irr-CE observed. As the irreversible reactions at the anode cannot be observed, this protocol acts as an efficient tool to extract information relating to the irreversible reactions at the cathode, including oxidative electrolyte decomposition (Ox. E.D.), cathode degradation, and first intrinsic irreversible capacity of cathode material (first irr-cap. of cathode) in the first cycle ${ }^{38-42}$. Generally, the irr-CE of cathode/Li cells in the first cycle is often found larger due to the initial Ox. E.D. and the correlated cathode-electrolyte interphase (CEI) formation than that in the subsequent cycles. In particular, the first irr-capacity of the cathode is significantly larger and often observed in layered oxide cathode materials, which can be attributed to mainly the slow lithium kinetics at high lithium contents and partially the formation of $\mathrm{Li}_{2} \mathrm{MO}_{2}$-like phases. This intrinsic irr-capacity cannot be recovered in the absence of deep discharge of the cell or unless cycling at the higher temperature to eliminate the kinetics limitation based on our own experiments and the previously reported works (Supplementary Fig. 3) ${ }^{38-40}$. Based on the above mentioned three origins of irr-CE at the cathode, we can dissect the irr-CEs of cathode/Li cell by first quantifying the first cycle irr-CE as the first irr-cap. of the cathode (with Ox. E.D. included, yellow bar in Fig. 3g). Second, in the subsequent cycles, the origins of irr-CEs can be separated into two sources. When the reversible capacity remains the same and stable, the irr-CE of the cell can be attributed to the subsequent oxidative electrolyte decomposition (Sub. Ox. E.D., blue bar in Fig. 3g) with the consequent CEI formation included; however, when the reversible capacity starts to fade, then the irr-CE would become the sum of cathode degradation (denoted as cathode degrad. and shown in gray bar in Fig. 3g) and sub. Ox. E.D. owing to the fact that capacity fading is directly related to cathode degradation. To be more specific, the fraction of cathode degradation can be calculated from the slope of the fitted line of the normalized discharged capacity retention based on equation (1) in the supporting information. Thus, the fraction of sub. Ox. E.D. within the capacity fading region could be finally quantified as the difference between the total irr-CE of cathode/Li cell and that of cathode degradation.

The cathode/Cu cell, which is also called AFLMB as displayed in Fig. 2d, can be recognized as a full cell with the $\mathrm{A} / \mathrm{C}$ ratio $\cong 1$ since the active Li is purely from the cathode. The two halfreactions taking place in the AFLMB are comparable to those in cathode/Li and $\mathrm{Li} / \mathrm{Cu}$ cells, namely $\mathrm{Li}$ deposition/dissolution on $\mathrm{Cu}$ and cathode oxidation/reduction, respectively. When the anode-free cell is fully charged, $\mathrm{Li}^{+}$is de-intercalated from NMC and plated onto the $\mathrm{Cu}$ along with the formation of dendritic or mossy $\mathrm{Li}$ (Fig. 3c). In reverse, $\mathrm{Li}^{+}$is stripped from the anode with some dead Li left on $\mathrm{Cu}$ and intercalated back into NMC (Fig. 3f). Therefore, the irreversible capacity and CE of AFLMB are significantly relative to that of $\mathrm{Li} / \mathrm{Cu}$ and cathode/Li cells, and by 


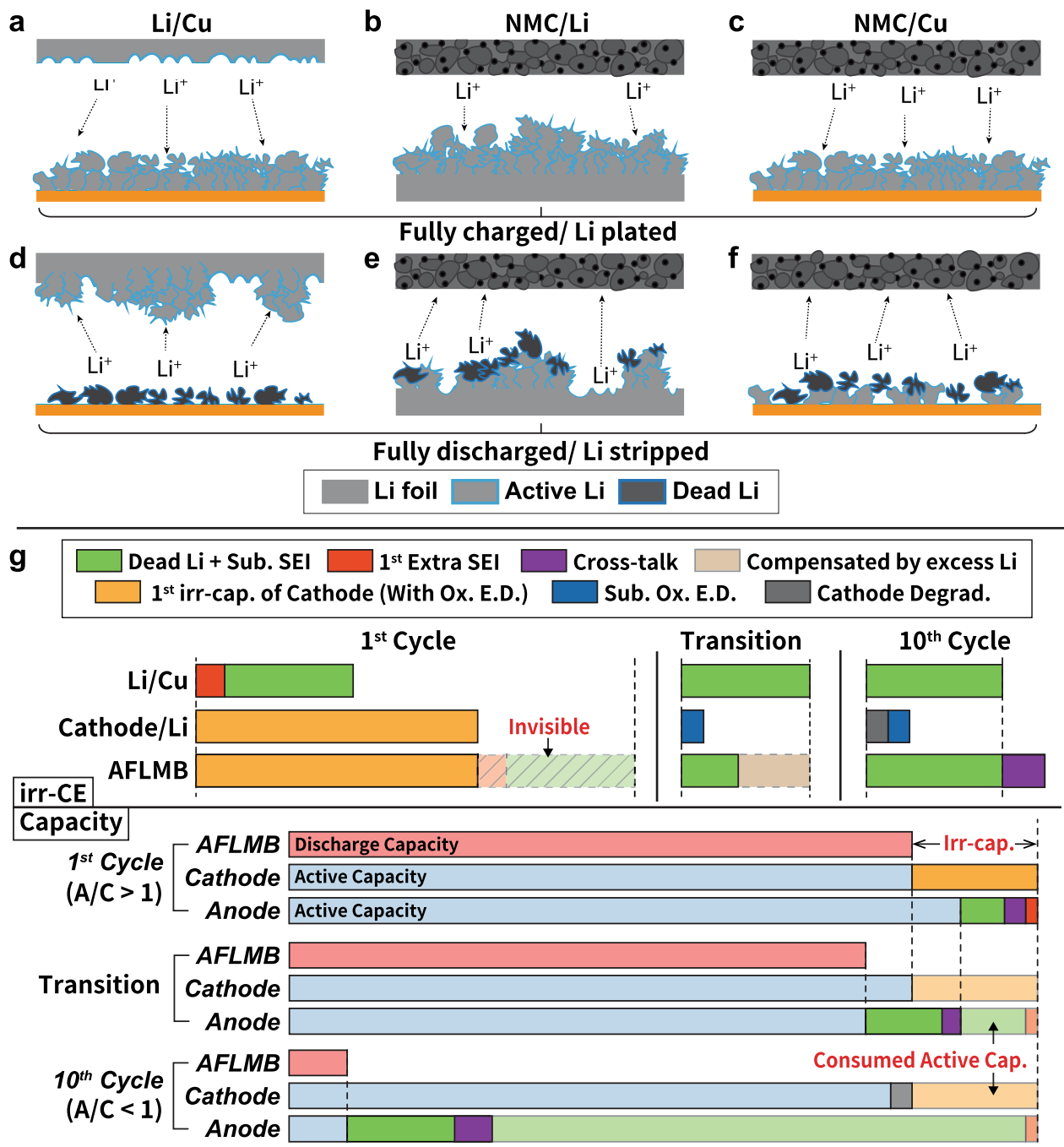

Fig. 3 Integrated protocol and scheme of different cell configurations at fully charged and discharged states. Scheme of a Li/Cu, $\mathbf{b} \mathrm{NMC} / \mathrm{Li}$, and c NMC/Cu cells at fully charged/ Li pated sate in the first cycle, respectively. Scheme of $\mathbf{d} \mathrm{Li} / \mathrm{Cu}$, e NMC/Li, and $\mathbf{f} \mathrm{NMC} / \mathrm{Cu}$ cells at fully discharge/Listripped state in the first cycle, respectively. $\mathbf{g}$ Proposed integrated protocol to unravel the origins of irreversible CE in AFLMB by Li/CU and cathode/Li cells. The blue shell on Li represents the SEI layer. Sub. SEI stands for the subsequent SEI formation, Ox. E.D. for oxidative electrolyte decomposition, and cathode degrad. for cathode degradation, respectively.

integrating the information of $\mathrm{Li} / \mathrm{Cu}$ and cathode/Li cells, it is possible to dissect the proportion of irreversible capacity and $\mathrm{CE}$ in the anode-free cell at its different $\mathrm{A} / \mathrm{C}$ ratio states generated from different origins as shown in Fig. $3 \mathrm{~g}$ and will be discussed in the following paragraphs. Nevertheless, it should be noted that apart from the sources of irr-CEs and capacity loss in $\mathrm{Li} / \mathrm{Cu}$ and cathode/Li cells would affect the evaluation of AFLMB; cross-talk effects would also account for the addition irr-CE upon the cycling of AFLMB. During the cycling of AFLMB, cross-talk effects could take place, namely the crossover of transition metal ions from the cathode materials to anode, leading to greatly altered Li plating/stripping chemistry as well as the SEI formation mechanism ${ }^{43,44}$. In other words, more complicated side reactions and higher irr-CE of the Li-plating/stripping processes than those in $\mathrm{Li} / \mathrm{Cu}$ cell may occur. Therefore, when dissecting the irr-CEs of AFLMB, cross-talk effects should also be considered. The detailed step-by-steps flowchart showing the procedures from identifying the irr-CEs in both $\mathrm{Li} / \mathrm{Cu}$ and cathode/Li cells and transferring those into AFLMB is provided in the Supplementary information (Supplementary Fig. 3).

As the irr-capacity of anode and cathode could changes along with the cycles in an AFLMB, its $\mathrm{A} / \mathrm{C}$ ratio and limiting electrode would also change, which would affect the analysis of the contribution of irr-CE. Therefore, it is important to know how the capacity changes in both electrodes. From the perspective of capacity, if the cathode material used possesses the first intrinsic irrcapacity of layered oxide cathode, excess active Li would be left on $\mathrm{Cu}$ in the form of metallic $\mathrm{Li}$ causing the $\mathrm{A} / \mathrm{C}$ ratio $>1$ and cathode as limiting electrode after the first cycle, causing the irr-CE of AFLMB only contributed from the cathode as that in an NMC/Li cell, namely first irr-capacity of cathode and sub. Ox. E.D. In contrast, when the $\mathrm{A} / \mathrm{C}$ ratio is $<1$, the irr-capacity of AFLMB would be only ascribed to the anode. To study the effect of A/C ratio on the capacity retention of (AF) LMBs, Supplementary Fig. 6 and Supplementary Fig. 7 show the charge/discharge profiles and cycling performance results of $\mathrm{NMC/Li}$ cells with different $\mathrm{A} / \mathrm{C}$ ratio. When the reversible capacity is kept almost the same as that of the first cycle discharge capacity as if in cathode/Li cell, it can be reckoned as the $\mathrm{A} / \mathrm{C}$ ratio is $>1$ where all the irr-capacity observed arouses from the cathode. (Supplementary Fig. 7b) However, the hidden chemistry beneath this phenomenon is the continued consumption of excess $\mathrm{Li}$. This is because dead Li forms in each cycle, yet the active Li inventory within AFLMB cells is solely provided by the cathode and limited. The active Li would be 
continuously consumed and eventually used up, as shown in Supplementary Fig. 7a. Thus, as the irr-capacity caused by the cathode degradation is significantly low compared with dead $\mathrm{Li}$ formation, the observed reversible (active) capacity of AFLMB can serve as an indicator of the $\mathrm{A} / \mathrm{C}$ ratio of the cell in situ.

During the continuous consumption of active Li inventory, there will be a transition state in a specific cycle when the A/C ratio is turning into $<1$. From Supplementary Fig. $7 \mathrm{~b}$, this unique circumstance can be characterized by a sudden fading slope transition of the discharge capacity of AFLMB, implying the excess $\mathrm{Li}$ is no longer enough to compensate the dead $\mathrm{Li}$ formation in each cycle, which leads to less active $\mathrm{Li}$ at the anode than that at the cathode, limiting the observed reversible capacity of AFLMB as shown in Fig. 3g. Therefore, the irr-CE observed will combine both anode and cathode in the transition state and dominated by dead $\mathrm{Li}$ and subsequent SEI formation afterward. To conclude, the larger the $\mathrm{A} / \mathrm{C}$ ratio and less severe dead $\mathrm{Li}$ formation, the later the transition state occurs as shown in Supplementary Fig. 7b. Thus, by enhancing the CE of Li plating/ stripping and suppressing dead $\mathrm{Li}$ formation, the capacity retention and the high CE region can be improved and prolonged in AFLMBs, respectively.

In view of irr-CE, the first cycle irr-CE of AFLMB can be explained by the sum of first SEI formation, dead $\mathrm{Li}+$ sub. SEI, and cross-talk effect when the initial A/C ratio is $<1$. The fraction of first SEI formation and dead $\mathrm{Li}+$ sub. SEI can be transferred from $\mathrm{Li} / \mathrm{Cu}$ cell, and by subtracting the value of total irr-CE with that of the aforementioned value in $\mathrm{Li} / \mathrm{Cu}$ cell, the fraction of cross-talk effects (purple bar in Fig. 3g) can be obtained. As in the subsequent cycles, the irr-CE of AFLMB will be the combination of dead Li+ sub. SEI and cross-talk effects. Meanwhile, when the initial A/C ratio is $>1$, namely cathode is the limiting electrode, then the first cycle irr-CE is dominated by cathode and equals to the fraction of the first irrcapacity of cathode in cathode/Li cell. In the subsequent cycles, the irr-CE can be separated into cathode degradation and sub. Ox. E.D. when $\mathrm{A} / \mathrm{C}$ ratio remains larger than one, namely before the transition state. However, the value of cathode degradation in AFLMB may not necessarily equal to that in cathode/Li cell considering the mechanism may be different among two cell configurations. Eventually, when the $\mathrm{A} / \mathrm{C}$ ratio becomes $<1$ after the transition state, the anode is then the limiting electrode and dominates the irr-CE of AFLMB, which is comparable to the aforementioned $\mathrm{A} / \mathrm{C}<1$ case that the irr-CE can be separated into cross-talk effects and dead $\mathrm{Li}+$ sub. SEI. Thus, considering the effect of $\mathrm{A} / \mathrm{C}$ ratio on irr- $\mathrm{CE}$, when the $\mathrm{A} / \mathrm{C}$ ratio is $>1$, the proportion of irr-CE from the anode is not observable and plotted in the light color bar with a dashed outline in Fig. $3 \mathrm{~g}$. When in the transition state, some of the irr-CE from dead $\mathrm{Li}$ formation will be compensated by excess $\mathrm{Li}$ as shown in the brown bar in Fig. $3 \mathrm{~g}$. At last, when the $\mathrm{A} / \mathrm{C}$ ratio is $<1$, the irr-CE of AFLMB dominated by dead $\mathrm{Li}$ and subsequent SEI formation.

Example 1: $1 \mathrm{M} \mathrm{LiPF}_{6}$ in EC:DEC under $0.2 \mathrm{~mA} \mathrm{~cm}^{-2}$. Figure 4 shows the corresponding irreversible $\mathrm{CE}$ at the first, the second, and the tenth cycle of $\mathrm{Li} / \mathrm{Cu}, \mathrm{NMC} / \mathrm{Li}$, and $\mathrm{NMC} / \mathrm{Cu}$ cells under the current density of $0.2 \mathrm{~mA} \mathrm{~cm}^{-2}$ in the commercial electrolyte ( $1 \mathrm{M}$ lithium hexafluorophosphate $\left(\mathrm{LiPF}_{6}\right)$ in EC:DEC). From the result obtained from the integrated protocol, the irr-CE of the first and the second cycle of $\mathrm{Li} / \mathrm{Cu}$ cell is $8.36 \%$ and $5.79 \%$, respectively, suggesting the $2.57 \%$ of higher irr-CE than second cycle is mainly caused by the first SEI formation, where the rest of that can be considered as dead $\mathrm{Li}+$ sub. SEI formation based on the protocol discussed in Fig. 3g. For cathode/Li cell (Fig. 2a) with A/C = 50, the irr-CE of $10.36 \%$ in first cycle can be ascribed to the first intrinsic irr-capacity of cathode with the corresponding Ox. E.D., and with that of $0.9 \%$ in the second cycle to subsequent Ox. E.D. In the subsequent cycles, the fraction of cathode degradation is successfully extracted and quantified as $0.076 \%$, with the rest of $0.36 \%$ attributed to subsequent oxidative electrolyte decomposition. In addition, it should be stated that the cycling performance of $\mathrm{NMC} / \mathrm{Li}$ is only independent of $\mathrm{Li}$ when the amount of active $\mathrm{Li}$ is sufficient enough with low charge/discharge rate. When increasing the current density to $0.4 \mathrm{~mA} \mathrm{~cm}^{-2}$, it is found that the capacity is declining quickly owing to the cathode degradation induced from soft short circuit and severe dendrite formation, as shown in Supplementary Fig. 9 and 10.

In the $\mathrm{NMC} / \mathrm{Cu}$ cell, the first cycle irr-CE of $\mathrm{NMC} / \mathrm{Cu}$ (AFLMB) cell is comparable to NMC/Li cell and can be described as the first irr-capacity of cathode, as the $\mathrm{A} / \mathrm{C}$ ratio is currently $>1$ and the cathode is the limiting electrode, making the irr-CE from anode invisible. In the second cycle, the irr-CE is decreased to $4.48 \%$, with the contribution mainly from dead $\mathrm{Li}+$ sub. SEI formation as the cell is in the transition state to $\mathrm{A} / \mathrm{C}<1$ and the irr-capacity and $\mathrm{CE}$ are limited by the anode. However, the higher irr-CE of AFLMB than $\mathrm{Li} / \mathrm{Cu}$ cell in the tenth cycle may be owing to more complicated cell chemistry due to cross-talk effect ${ }^{43-45}$. Thus, the irr-CE can be separated into $5.67 \%$ of dead $\mathrm{Li}+$ sub. SEI formation and $8.59 \%$ of cross-talk effects, respectively. Yet, it can still be concluded that dead Li formation is undoubtedly the most significant obstacle currently for the development of highperformance AFLMBs. In addition, experimental validation for the results obtained from the protocol is performed by measuring the amount of inactive $\mathrm{Li}$ in both $\mathrm{Li} / \mathrm{Cu}$ and $\mathrm{NMC} / \mathrm{Cu}$ cells at different cycles (Supplementary Discussion and Supplementary Fig. 5). It is found that the fraction of first extra SEI extracted from the protocol is very close to the practical SEI value measured from the TGC method. Moreover, the fraction of inactive $\mathrm{Li}^{+}$in the $\mathrm{NMC} / \mathrm{Cu}$ cell is found larger than that in $\mathrm{Li} / \mathrm{Cu}$ cell from the first cycle, confirming the fraction of SEI in $\mathrm{NMC/Cu}$ cell is affected by cross-talk effects, thus, larger than that in $\mathrm{Li} / \mathrm{Cu}$ cell. To conclude, the results of TGC measurements confirm the validity of our proposed protocol on dissecting the irr-CEs in both $\mathrm{Li} / \mathrm{Cu}$ and cathode/ $\mathrm{Cu}$ cells.

Example 2: $1 \mathrm{M} \mathrm{LiPF}_{6}$ in EC:DEC under $0.4 \mathrm{~mA} \mathrm{~cm}^{-2}$. The effect of current density is also studied using the integrated protocol. Figure 5 shows the obtained results using the same electrolyte from the previous section but under a current density of $0.4 \mathrm{~mA} \mathrm{~cm}^{-2}$. The first, second, and tenth cycle irr-CE of $\mathrm{Li} / \mathrm{Cu}$ cell is $7.5 \%, 6.2 \%$, and $8.6 \%$, respectively, representing $1.3 \%$ of first SEI formation on $\mathrm{Cu}$ with $6.2 \%$ of dead $\mathrm{Li}+$ sub. SEI formation in the first cycle and mainly owing to dead Li+ sub. SEI formation in the subsequent cycles. Although the irr-CE of $11.19 \%$ for $\mathrm{NMC} / \mathrm{Li}$ cell in the first cycle can be attributed to the first irr-capacity of NMC and that of $0.57 \%$ in the second cycle to subsequent oxidative electrolyte decomposition. As for the cathode degradation in the subsequent cycles, the fraction has been identified and calculated as $0.1 \%$, which is larger than that in 0.2 $\mathrm{mA} \mathrm{cm}{ }^{-2}$ case owing to the higher degradation rate under higher current density. Finally, after integrating the results from $\mathrm{Li} / \mathrm{Cu}$ and $\mathrm{NMC} / \mathrm{Li}$ cells, the irr-CE of $\mathrm{NMC/Cu}$ cell in the first cycle can be summarized as $11.68 \%$ first intrinsic irr-capacity of NMC as $\mathrm{A} / \mathrm{C}$ ratio is larger than one and the determining electrode is the cathode. In the second cycle, the irr-CE is significantly larger than that in $0.2 \mathrm{~mA} \mathrm{~cm}^{-2}$. This can be explained by the increased dendritic and dead $\mathrm{Li}$ formation rate and more serious SEI fracture under higher current density. In particular, the fraction of cross-talk effects is determined by subtracting the total irr-CE with that of $\mathrm{Li} / \mathrm{Cu}$ cell in the second cycle as $4.04 \%$. To conclude, the contribution of dead $\mathrm{Li}+$ sub. SEI formation in irr-CE is 

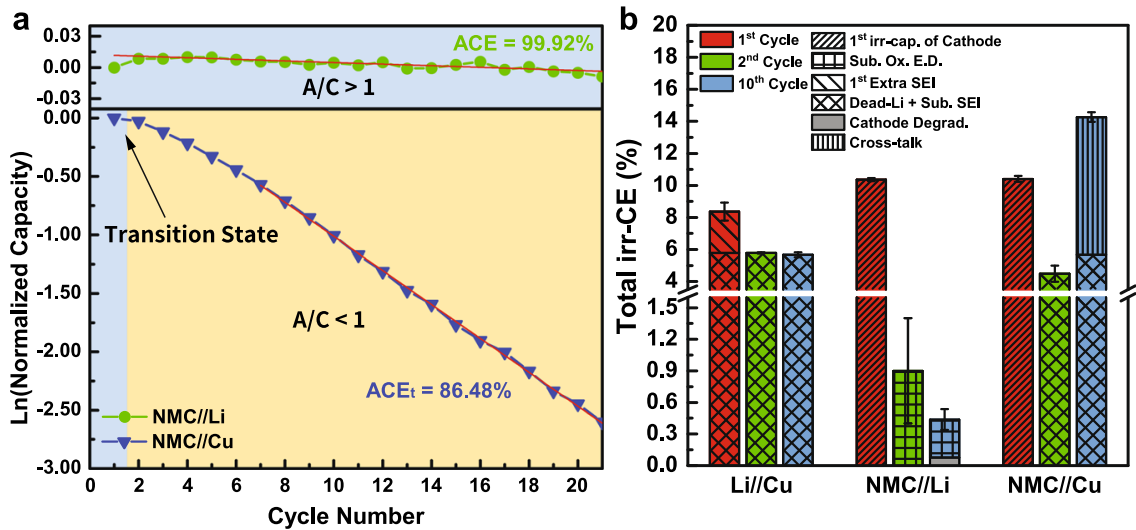

Fig. 4 Results obtained from integrated protocol using $\mathbf{1} \mathbf{M ~ L i P F}_{6}$ in EC:DEC (1:1) as electrolyte under the current density of $\mathbf{0 . 2} \mathbf{m A ~} \mathrm{cm}^{-2}$. a Normalized discharge capacity versus cycle number of NMC/Li and NMC/Cu cells. $\mathbf{b}$ irreversible CE comparison of $\mathrm{Li} / \mathrm{Cu}, \mathrm{NMC} / \mathrm{Li}$, and NMC/Cu cells. Error bars represent standard deviation, $n=3$ independent replicates. The capacity retention comparison of NMC/Li and NMC/Cu cells are shown in Supplementary Fig. 8 a.
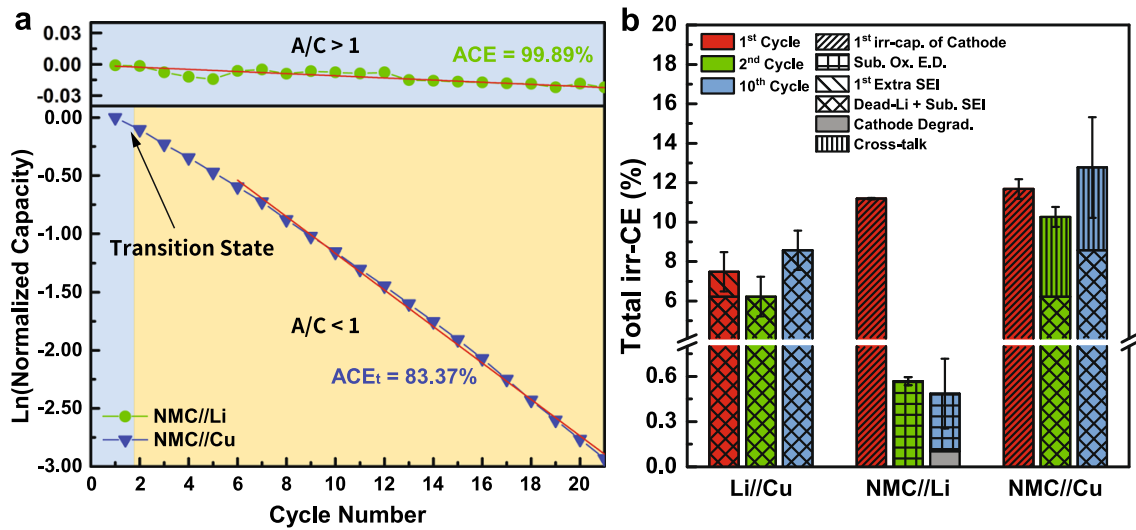

Fig. 5 Results obtained from integrated protocol using $1 \mathrm{M} \mathrm{LiPF}_{6}$ in EC:DEC (1:1) as electrolyte under the current density of $0.4 \mathrm{~mA} \mathrm{~cm}^{-2}$. a Normalized discharge capacity versus cycle number of NMC/Li and NMC/Cu cells. $\mathbf{b}$ irreversible CE comparison of $\mathrm{Li} / \mathrm{Cu}, \mathrm{NMC} / \mathrm{Li}$, and NMC/Cu cells. Error bars represent standard deviation, $n=3$ independent replicates. The capacity retention comparison of NMC/Li and NMC/Cu cells are shown in Supplementary Fig. 8b. The charge/discharge profiles of each cell configuration are shown in Supplementary Fig. 12.
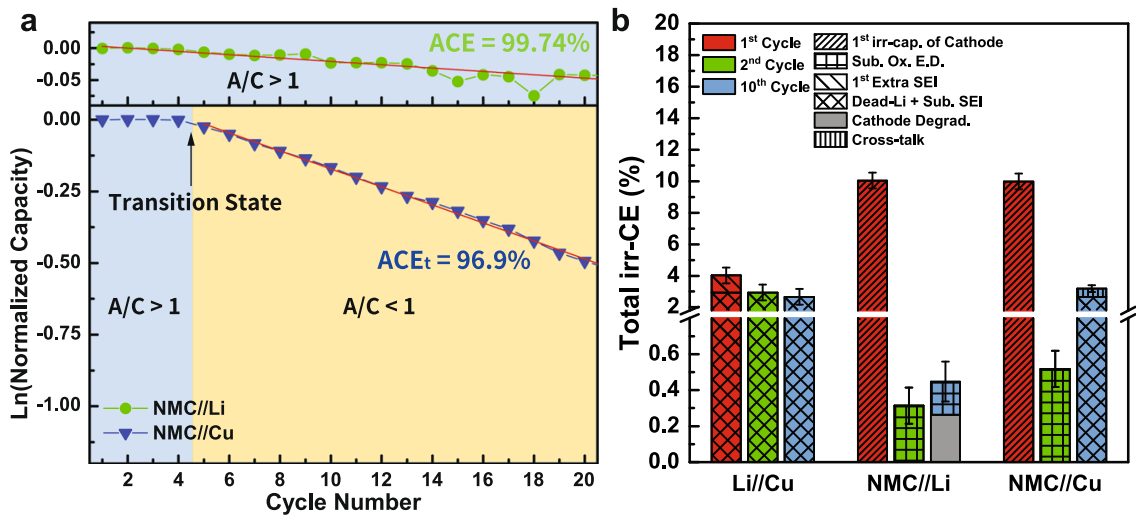

Fig. 6 Results obtained from integrated protocol using $1 \mathrm{M} \mathrm{LiPF}_{6}$ in EC:DEC (1:1) with 5\% FEC added as electrolyte under the current density of 0.2 $\mathbf{m A ~} \mathbf{~ c m}^{-\mathbf{2}}$. a Normalized discharge capacity versus cycle number of NMC/Li and NMC/Cu cells. $\mathbf{b}$ irreversible CE comparison of Li/Cu, NMC/Li, and $\mathrm{NMC} / \mathrm{Cu}$ cells. Error bars represent standard deviation, $n=3$ independent replicates. The capacity retention comparison of NMC/Li and NMC/Cu cells are shown in Supplementary Fig. 8c. The charge/discharge profiles of each cell configuration are shown in Supplementary Fig. 13.

higher and consumes more amount of excess Li in each cycle, causing the faster transition to $\mathrm{A} / \mathrm{C}<1$ and higher irr- $\mathrm{CE}$ of the second cycle than $0.2 \mathrm{~mA} \mathrm{~cm}^{-2}$, and the major sources of irr-CE in AFLMB are still originated from the anode, namely dead $\mathrm{Li}+$ sub. SEI formation and cross-talk effects.
Example 3: $1 \mathrm{M} \mathrm{LiPF}_{6}$ in EC:DEC with 5\% FEC. Figure 6 shows the results obtained from the integrated protocol using $1 \mathrm{M} \mathrm{LiPF}_{6}$ in EC:DEC with 5\% FEC as the electrolyte under the current density of $0.2 \mathrm{~mA} \mathrm{~cm}^{-2}$. The first and second cycle irr-CE of Li/ $\mathrm{Cu}$ is $4.02 \%$ and $2.94 \%$, respectively. Similar to the previous case, 
the $1.08 \%$ difference can be attributed to the first SEI formation on $\mathrm{Cu}$, and the rest of $2.94 \%$ can be attributed to dead $\mathrm{Li}+$ sub. SEI formation. From the result of $\mathrm{Li} / \mathrm{Cu}$ cell, the effect of FEC on suppressing dead $\mathrm{Li}$ and dendrite formation can already be revealed from the lower irr-CE than that of without FEC additive. Meanwhile, the irr-CE of $\mathrm{NMC/Li}$ cell in first cycle can be explained as the first intrinsic irr-capacity of NMC with the corresponding oxidative electrolyte decomposition (10.04\%) and that in second cycle as subsequent oxidative electrolyte decomposition $(0.31 \%)$. Meanwhile, the fraction of cathode degradation is determined as $0.26 \%$ from equation (1) in the supporting information, with the rest of $0.19 \%$ ascribing to electrolyte decomposition. Finally, the irr-CE of $\mathrm{NMC} / \mathrm{Cu}$ in the first cycle, same as that of $\mathrm{NMC} / \mathrm{Li}$ cell, can be attributed to $9.98 \%$ of the first intrinsic irr-capacity of NMC with oxidative electrolyte decomposition when the $\mathrm{A} / \mathrm{C}$ ratio $>1$. However, the irr- $\mathrm{CE}$ of the second cycle is $0.52 \%$ and significantly lower than that in the $\mathrm{NMC} / \mathrm{Cu}$ cell without FEC added. This can be again explained by the effect of FEC suppressing dead $\mathrm{Li}+$ sub. SEI formation, leading to slower consumption of excess active $\mathrm{Li}$ on $\mathrm{Cu}$. Thus, the $\mathrm{A} / \mathrm{C}$ ratio of the cell is sustained greater than one longer than that without FEC additive before transiting to $\mathrm{A} / \mathrm{C}<1$, and the irr-CE of the first four cycles is very low, as shown in Fig. 6a. In other words, the transition state is delayed to the fifth cycle. Meanwhile, the discharge capacity is found without decaying in the first four cycles, which also proves that the $\mathrm{A} / \mathrm{C}>1$ region is extended to four cycles. As a result, the irr-CEs from the second to fourth cycle can be determined as subsequent oxidative electrolyte decomposition at the cathode like in the $\mathrm{NMC/Li}$ cell, different from those without FEC added. Later on, after the transition state and the $\mathrm{A} / \mathrm{C}$ becomes $<1$, the increased irr-CE of $3.18 \%$ can be thus attributed to mainly dead $\mathrm{Li}+$ sub. SEI formation $(2.66 \%)$ and partially cross-talk effects $(0.52 \%)$ at the anode.

To conclude, if we compare the results obtained from the proposed protocol between with/without FEC as an additive, the lower initial overpotential and polarization shown by $\mathrm{Li} / \mathrm{Li}$ and $\mathrm{Li} / \mathrm{Cu}$ cells after the introduction of FEC reveals the effect of FEC on forming better SEI and favoring $\mathrm{Li}$ nucleation on $\mathrm{Cu}$. The lower irr-CE of $\mathrm{Li} / \mathrm{Cu}$ cell and significantly lower irr-CE after the first cycle in $\mathrm{NMC/Cu}$ cell indicate the ability of FEC to suppress dendrite and dead Li. Interestingly, the irr-CE of NMC/Li cell does not show a significant difference with/without FEC, suggesting the contribution of FEC is less notable for the cathode. Although it has been reported that the addition of FEC into electrolytes can lead to a more compacted and stable LiF-rich SEI, which is also beneficial to the uniform $\mathrm{Li}$ deposit and better electrochemical performance ${ }^{46}$. By summarizing all the contributions from the above configuration within the proposed protocol, we can quantitively unravel the effects of FEC on mitigating the formation of dead $\mathrm{Li}$ and forming stable SEI in AFLMB.

\section{Discussion}

In summary, we revealed the formation of dead $\mathrm{Li}$ and dendritic $\mathrm{Li}$ alongside the ISC via in situ OM and proposed the mechanism of $\mathrm{Li}$ deposition/dissolution on $\mathrm{Cu}$. Furthermore, by combining the information and irr-CE of four different cell set-ups, an integrated protocol is proposed to unravel the concealed messages of various irr-CE. From the demystified information, the origin and proportion of irreversible coulombic efficiency in AFLMBs induced from first extra SEI, dead Li and subsequent SEI, crosstalk effects, first cycle intrinsic irreversible capacity of cathode, and subsequent oxidative electrolyte decomposition can be quantified and revealed. Although AFLMBs suffer from high
irr-CE and quick capacity fading and are often recognized as a poor cell configuration, AFLMB could also serve as an indispensable key in facilitating the development of better electrolytes and evaluating the performance of LMBs. In general, the integrated protocol proposed here can be further expanded to comprehensively examine the effectiveness of various strategies on improving the electrochemical performance of LMBs or AFLMBs, providing an insightful understanding of the behaviors of LMBs and AFLMBs and paving the way to the realization of nextgeneration high-energy rechargeable lithium batteries involving metal deposition/stripping chemistry.

\section{Methods}

Materials. The commercial NMC electrodes (Amita Technologies Inc. Taiwan) contain $88.5 \mathrm{wt} \%$ of active material $\left(\mathrm{LiNi}_{1 / 3} \mathrm{Co}_{1 / 3} \mathrm{Mn}_{1 / 3} \mathrm{O}_{2}, \mathrm{NMC}-111\right), 4 \mathrm{wt} \%$ of polyvinylidene fluoride binder, and $7.5 \mathrm{wt} \%$ of conductive carbon $(2.5 \mathrm{wt} \%$ of Super $\mathrm{P}$ with $5 \mathrm{wt} \%$ of KS6). The nominal areal discharge capacity of NMC electrodes used in this work is $2 \mathrm{mAh} \mathrm{cm}^{-2}$.

$\mathrm{Cu}$ foil was used as an anode electrode in the anode-free cell. The $\mathrm{Cu}$ foil was cut into $19 \mathrm{~mm}$ in diameter, washed by $1 \mathrm{M}$ hydrochloric acid for $10 \mathrm{~min}$ in an ultrasonic cleaner, followed by rinsing with deionized water and acetone three times, and finally vacuumed in the desiccator for $30 \mathrm{~min}$ before use.

Cell assembly. The $\mathrm{OM} \mathrm{Li} / \mathrm{Cu}$ cell was assembled by sealing a $\mathrm{Li}$ foil and copper foil at a distance $\sim 250 \mu \mathrm{m}$ in-between inside a plastic pouch cell. Copper wire was used as a terminal for both electrodes and the external circuit for electrochemical measurements. The OM cells and CR2032 coin cells were all assembled in an argon-filled glovebox (UNIlab Plus Glove Box, MBRAUN) where the oxygen and moisture content were kept $<1 \mathrm{ppm}$.

Li foil ( $\sim 300 \mu \mathrm{m}$, FMC Corporation) was attached on a $500 \mu \mathrm{m}$ spacer and used as an anode in $\mathrm{NMC/Li}$ cell, identical electrodes in $\mathrm{Li} / \mathrm{Li}$ symmetric cell, and trilayer Celgard $2325 \mathrm{PP} / \mathrm{PE} / \mathrm{PP}$ membrane as a separator. The $\mathrm{NMC} / \mathrm{Cu}$ cells were assembled by pairing NMC electrode with a copper foil, with an $800 \mu \mathrm{m}$ spacer to minimize the thickness difference of assemblies within the cell between $\mathrm{NMC} / \mathrm{Cu}$ and NMC/Li cells to unify the cell pressure and make the results obtained from each cell configuration the most reliable. The electrolyte used was $1 \mathrm{M} \mathrm{LiPF}_{6}$ in the mixture of ethylene carbonate (EC) and diethyl carbonate (v:v=1:1) (SigmaAldrich) with or without $5 \%$ of fluoroethylene carbonate.

Dead Li quantification. Dead Li quantification was performed using TGC method reported by Meng et al. ${ }^{32}, \mathrm{Li} / \mathrm{Cu}$ cells at their stripped state were first disassembled inside an Ar-filled glovebox $\left(\mathrm{H}_{2} \mathrm{O}, \mathrm{O}_{2}<1\right.$ p.p.m.), then the $\mathrm{Cu}$ foil with residual inactive $\mathrm{Li}$ and separator were transferred into a $20 \mathrm{ml}$ vial. The vial was sealed by a plastic lid with a rubber septum in the middle, then tightly wrapped with parafilm to prevent any gas leakage when $\mathrm{H}_{2}$ gas was generated later. The pressure inside the vial was adjusted to $1 \mathrm{~atm}(0 \mathrm{mbar}$ inside the glovebox environment) before the sealing process. After transferring the vial out of the glovebox, $0.5 \mathrm{ml}$ of water was injected into the vial to allow a reaction with dead Li. The added excess amount of water would fully react with inactive metallic $\mathrm{Li}$, generating lithium hydroxide and $\mathrm{H}_{2}$ gas. After complete reaction and $\mathrm{H}_{2}$ gas formation, a gas-tight syringe was used to take $250 \mu \mathrm{l}$ of gas within the vial and to inject it into the GC for $\mathrm{H}_{2}$ measurement. The calibration line of GC was measured by weighing several different weights of metallic $\mathrm{Li}$ and measured the respective $\mathrm{H}_{2}$ areas as a function.

In situ OM observation. In situ plating and stripping of Li on copper foil was observed using OM in a plastic pouch cell by applying $500 \mathrm{~mA} \mathrm{~cm}^{-2}$ of charge/ discharge current density, as shown in Fig. 1a and the movies are also included in the supporting information. The electrochemical measurement of the cell was simultaneously conducted on a PGSTAT101 Autolab potentiostat (Metrohm), with the potential and current resolution of $3 \mu \mathrm{V}$ and $10 \mathrm{nA}$, respectively.

Electrochemical tests. Galvanostatic charge/discharge was used to cycle batteries with an applied current density of either 0.2 or $0.4 \mathrm{~mA} \mathrm{~cm}^{-2}$ to study the effect of charge/discharge rate. Galvanostatic charge/discharge tests were performed on Arbin BT-2000 (Arbin Instruments) battery test equipment at room temperature.

\section{Data availability}

The data that support the findings of this study are available from the authors on reasonable request. Correspondence and requests for materials should be addressed to B.-J.H.

Received: 18 July 2020; Accepted: 1 February 2021; Published online: 04 March 2021 


\section{References}

1. Armand, M. \& Tarascon, J. M. Building better batteries. Nature 451, 652-657 (2008).

2. Tarascon, J. M. \& Armand, M. Issues and challenges facing rechargeable lithium batteries. Nature 414, 359-367 (2001).

3. Li, S. et al. Developing high-performance lithium metal anode in liquid electrolytes: challenges and progress. Adv. Mater. 30, e1706375 (2018).

4. Cheng, J.-H. et al. Visualization of lithium plating and stripping via in operando transmission X-ray microscopy. J. Phys. Chem. C. 121, 7761-7766 (2017).

5. Jiao, S. et al. Behavior of lithium metal anodes under various capacity utilization and high current density in lithium metal batteries. Joule $\mathbf{2}$, 110-124 (2018).

6. Liu, B., Zhang, J.-G. \& Xu, W. Advancing lithium metal batteries. Joule 2 , 833-845 (2018)

7. $\mathrm{Xu}, \mathrm{W}$. et al. Lithium metal anodes for rechargeable batteries. Energy Environ. Sci. 7, 513-537 (2014)

8. Lin, D., Liu, Y. \& Cui, Y. Reviving the lithium metal anode for high-energy batteries. Nat. Nanotechnol. 12, 194-206 (2017).

9. Cheng, X. B., Zhang, R., Zhao, C. Z. \& Zhang, Q. Toward safe lithium metal anode in rechargeable batteries: a review. Chem. Rev. 117, 10403-10473 (2017).

10. Haregewoin, A. M., Wotango, A. S. \& Hwang, B.-J. Electrolyte additives for lithium ion battery electrodes: progress and perspectives. Energy Environ. Sci. 9, 1955-1988 (2016).

11. Zhang, Y. et al. Towards better Li metal anodes: challenges and strategies. Mater. Today 33, 56-74 (2020).

12. Ryou, M.-H., Lee, Y. M., Lee, Y., Winter, M. \& Bieker, P. Mechanical surface modification of lithium metal: towards improved Li metal anode performance by directed Li plating. Adv. Funct. Mater. 25, 834-841 (2015).

13. Zheng, J. et al. Highly stable operation of lithium metal batteries enabled by the formation of a transient high-concentration electrolyte layer. Adv. Energy Mater. 6, 1502151 (2016)

14. Tripathi, A. M., Su, W. N. \& Hwang, B. J. In situ analytical techniques for battery interface analysis. Chem. Soc. Rev. 47, 736-851 (2018).

15. Choi, J. W. \& Aurbach, D. Promise and reality of post-lithium-ion batteries with high energy densities. Nat. Rev. Mater. 1, 1-16 (2016).

16. $\mathrm{Xu}, \mathrm{R}$. et al. Artificial interphases for highly stable lithium metal anode. Matter 1, 317-344 (2019).

17. Pang, Q., Liang, X., Shyamsunder, A. \& Nazar, L. F. An in vivo formed solid electrolyte surface layer enables stable plating of Li metal. Joule 1, 871-886 (2017).

18. Hagos, T. T. et al. Developing high-voltage carbonate-ether mixed electrolyte via anode-free cell configuration. J. Power Sources 461, 228053 (2020).

19. Zhang, Y. et al. A 3D lithium/carbon fiber anode with sustained electrolyte contact for solid-state batteries. Adv. Energy Mater. 10, 1903325 (2019).

20. Wang, S. H. et al. Tuning wettability of molten lithium via a chemical strategy for lithium metal anodes. Nat. Commun. 10, 4930 (2019).

21. Chen, S. et al. Critical parameters for evaluating coin cells and pouch cells of rechargeable Li-metal batteries. Joule 3, 1094-1105 (2019).

22. Wang, J. et al. Improving cyclability of Li metal batteries at elevated temperatures and its origin revealed by cryo-electron microscopy. Nat. Energy 4, 664-670 (2019).

23. Louli, A. J., Ellis, L. D. \& Dahn, J. R. Operando pressure measurements reveal solid electrolyte interphase growth to rank Li-ion cell performance. Joule 3 , 745-761 (2019).

24. Niu, C. et al. High-energy lithium metal pouch cells with limited anode swelling and long stable cycles. Nat. Energy 4, 551-559 (2019).

25. Liu, J. et al. Pathways for practical high-energy long-cycling lithium metal batteries. Nat. Energy 4, 180-186 (2019).

26. Qian, J. et al. Anode-free rechargeable lithium metal batteries. Adv. Funct. Mater. 26, 7094-7102 (2016).

27. Hagos, T. M. et al. Dual electrolyte additives of potassium hexafluorophosphate and tris (trimethylsilyl) phosphite for anode-free lithium metal batteries. Electrochim. Acta 316, 52-59 (2019).

28. Beyene, T. T. et al. Concentrated dual-salt electrolyte to stabilize Li metal and increase cycle life of anode free Li-metal batteries. J. Electrochem. Soc. 166, A1501-A1509 (2019).

29. Assegie, A. A. et al. Multilayer-graphene-stabilized lithium deposition for anode-Free lithium-metal batteries. Nanoscale 11, 2710-2720 (2019).

30. Weber, R. et al. Long cycle life and dendrite-free lithium morphology in anode-free lithium pouch cells enabled by a dual-salt liquid electrolyte. Nat. Energy 4, 683-689 (2019).

31. Hagos, T. T. et al. Locally concentrated LiPF6 in a carbonate-based electrolyte with fluoroethylene carbonate as a diluent for anode-free lithium metal batteries. ACS Appl Mater. Interfaces 11, 9955-9963 (2019).

32. Fang, C. et al. Quantifying inactive lithium in lithium metal batteries. Nature 572, 511-515 (2019).
33. Burns, J. C. et al. Introducing symmetric Li-ion cells as a tool to study cell degradation mechanisms. J. Electrochem. Soc. 158, A1417-A1422 (2011).

34. Thirumalraj, B. et al. Nucleation and growth mechanism of lithium metal electroplating. J. Am. Chem. Soc. 141, 18612-18623 (2019).

35. Gao, J. et al. Lithiated Nafion as polymer electrolyte for solid-state lithium sulfur batteries using carbon-sulfur composite cathode. J. Power Sources 382, 179-189 (2018).

36. Assegie, A. A., Cheng, J. H., Kuo, L. M., Su, W. N. \& Hwang, B. J. Polyethylene oxide film coating enhances lithium cycling efficiency of an anode-free lithium-metal battery. Nanoscale 10, 6125-6138 (2018).

37. Abrha, L. H. et al. Li7La2.75Ca0.25Zr1.75Nb0.25O12@LiClO4 composite film derived solid electrolyte interphase for anode-free lithium metal battery. Electrochim. Acta 325, 134825 (2019).

38. Kang, S.-H., Yoon, W.-S., Nam, K.-W., Yang, X.-Q. \& Abraham, D. P. Investigating the first-cycle irreversibility of lithium metal oxide cathodes for Li batteries. J. Mater. Sci. 43, 4701-4706 (2008).

39. Genovese, M., Louli, A. J., Weber, R., Hames, S. \& Dahn, J. R. Measuring the coulombic efficiency of lithium metal cycling in anode-free lithium metal batteries. J. Electrochem. Soc. 165, A3321-A3325 (2018)

40. Zhou, H., Xin, F., Pei, B. \& Whittingham, M. S. What limits the capacity of layered oxide cathodes in lithium batteries? ACS Energy Lett. 4, 1902-1906 (2019).

41. Teshager, M. A. et al. In situ DRIFTS analysis of solid-electrolyte interphase formation on $\mathrm{Li}$-Rich $\mathrm{Li} 1.2 \mathrm{Ni} 0.2 \mathrm{Mn} 0.6 \mathrm{O} 2$ and $\mathrm{LiCoO} 2$ cathodes during oxidative lectrolyte decomposition. ChemElectroChem 3, 337-345 (2016).

42. Lin, M.-H. et al. Revealing the mitigation of intrinsic structure transformation and oxygen evolution in a layered $\mathrm{Li} 1.2 \mathrm{Ni} 0.2 \mathrm{Mn} 0.6 \mathrm{O} 2$ cathode using restricted charging protocols. J. Power Sources 359, 539-548 (2017).

43. Betz, J. et al. Cross talk between transition metal cathode and Li metal anode: unraveling its influence on the deposition/dissolution behavior and morphology of lithium. Adv. Energy Mater. 9, 1900574 (2019).

44. Zhang, X.-Q. et al. Crosstalk shielding of transition metal ions for long cycling lithium-metal batteries. J. Mater. Chem. A 8, 4283-4289 (2020).

45. Lee, $H$. et al. Detrimental effects of chemical crossover from the lithium anode to cathode in rechargeable lithium metal batteries. ACS Energy Lett. 3 , 2921-2930 (2018)

46. Zhang, X.-Q., Cheng, X.-B., Chen, X., Yan, C. \& Zhang, Q. Fluoroethylene carbonate additives to render uniform li deposits in lithium metal batteries. Adv. Funct. Mater. 27, 1605989 (2017).

\section{Acknowledgements}

Financial support from the Ministry of Science and Technology of Taiwan (MOST 1092639-E-011-001-ASP, 109-2923-E-011-008, 109-3116-F-011-CC1, 109-2124-M-002-008, 109-2923-E-011-009, 109-2221-E-011-063-MY3), the Ministry of Education of Taiwan (U2RSC program, MOE 1080059, Taiwan's Deep Decarbonization Pathways toward a Sustainable Society Project (AS-KPQ-106- DDPP) from Academia Sinica as well as the supporting facilities from National Taiwan University of Science and Technology (NTUST) and National Synchrotron Radiation Research Centre (NSRRC) are all gratefully acknowledged.

\section{Author contributions}

C.-J.H. and B.J.H. conceived and designed the work; C.-J.H., B.T., H.-C.T., K.N.S., H.S. T.T.H., T.T.B., and L.-M.K. performed the experiments; C.-J.H., B.T., H.-C.T., H.S., T.T.H., T.T.B., L.-M.K., W.-N.S., S.-H.W., and B.J.H. analyzed the data; C.-C.W. helped measure and process the TXM data; C.-J.H. and B.J.H. wrote the paper. All the authors discussed the results and commented on the manuscript. All authors have approved the final version of the manuscript.

\section{Competing interests}

The authors declare no competing interests.

\section{Additional information}

Supplementary information The online version contains supplementary material available at https://doi.org/10.1038/s41467-021-21683-6.

Correspondence and requests for materials should be addressed to B.J.H.

Peer review information Nature Communications thanks the anonymous reviewer(s) for their contribution to the peer review of this work. Peer reviewer reports are available.

Reprints and permission information is available at http://www.nature.com/reprints

Publisher's note Springer Nature remains neutral with regard to jurisdictional claims in published maps and institutional affiliations. 
(c) (i) Open Access This article is licensed under a Creative Commons Attribution 4.0 International License, which permits use, sharing, adaptation, distribution and reproduction in any medium or format, as long as you give appropriate credit to the original author(s) and the source, provide a link to the Creative Commons license, and indicate if changes were made. The images or other third party material in this article are included in the article's Creative Commons license, unless indicated otherwise in a credit line to the material. If material is not included in the article's Creative Commons license and your intended use is not permitted by statutory regulation or exceeds the permitted use, you will need to obtain permission directly from the copyright holder. To view a copy of this license, visit http://creativecommons.org/ licenses/by/4.0/.

(C) The Author(s) 2021 